\title{
Follow-up study of detachment of the retinal pigment epithelium*
}

\author{
MICHAEL L. KLEIN, ${ }^{1}$ HANNA OBERTYNSKI, ${ }^{2}$ ARNALL PATZ, ${ }^{2}$ \\ STUART L. FINE, ${ }^{2}$ AND MOHANDAS KINI ${ }^{3}$ \\ From the ${ }^{1}$ Department of Ophthalmology, Good Samaritan Hospital and Medical Center, \\ and the University of Oregon Health Sciences Center, Portland; ${ }^{2}$ the Retinal Vascular Service, \\ Wilmer Institute, Johns Hopkins University School of Medicine, Baltimore; and the \\ ${ }^{3}$ Boston University Medical Center
}

SUMmaRY To determine the clinical course of untreated retinal pigment epithelial detachment we reviewed data from 50 eyes of 42 patients followed up for an average of 29 months without photocoagulation. In 14 eyes with avascular lesions, in patients under 50 years of age, all had a final vision of $20 / 30$ or better. None developed neovascularisation or haemorrhage. In 17 eyes with avascular lesions in patients aged over 50, $8(47 \%)$ flattened spontaneously. Five $(24 \%)$ developed neovascularisation, 2 of which became haemorrhagic, with vision of less than 20/200. In 19 senile eyes with vascular lesions $14(74 \%)$ had a final vision of $20 / 200$ or less. The prognosis in eyes with pigment epithelial detachments is related to the age of the patient and the presence of choroidal neovascularisation. Photocoagulation is seldom needed for pigment epithelial detachments in young patients. Its value in senile eyes is unproved.

Detachment of the pigment epithelium results from the accumulation of fluid derived from the choriocapillaris, either directly or from new vessels extending into the subpigment epithelial space. ${ }^{1}$ While photocoagulation has been used to flatten these lesions, ${ }^{2-4}$ their course when left untreated has not been well documented. We reviewed data from untreated eyes with pigment epithelial detachments which were studied with fundus photography, fluorescein angiography, and frequent follow-up examinations.

\section{Materials and methods}

Fluorescein angiograms performed at the Wilmer Institute and the Boston University Medical Center during the years 1968 through 1974 were reviewed. All those with avascular serous detachments of the pigment epithelium in the macula, as described by Gass, ${ }^{1}$ were selected. Also chosen were all eyes with pigment epithelial detachments accompanied by choroidal neovascularisation ${ }^{5}$ in * Read in part before the annual meeting of the American
Medical Association, San Francisco, 20 June 1977.

Correspondence to Dr Michael L. Klein, $1200 \mathrm{~N}$ W 23rd, Portland, Oregon 97210, USA. patients over 50 years of age. Information was then obtained from the patient's clinical records.

Fifty eyes of 42 patients met the following criteria and are the subject of this report: (1) clinical and fluorescein angiographic evidence of pigment epithelial detachment; (2) good quality fluorescein angiogram; (3) no photocoagulation treatment; and (4) minimum follow-up period of 6 months.

Eyes included in this study were not subjected to photocoagulation for one of the following reasons: (1) evaluation took place prior to 1970 , which is when laser photocoagulation was first employed in the treatment of macular disorders at the Wilmer Institute; (2) evaluation was performed at the Wilmer Institute subsequent to 1973 , when a strictly conservative treatment approach was adopted; (3) the patient was evaluated at the Boston University Medical Center as part of a study of the natural history of macular diseases; or (4) the patient was under the care of an ophthalmologist who did not feel photocoagulation was of any benefit in this disorder.

Details of the initial examination, along with clinical and angiographic features of the lesion, were recorded. This included visual acuity, size of pigment epithelial elevation (disc diameters), distance from the fovea (defined as the centre of the retinal 
avascular zone on fluorescein angiography), and the presence of sensory retinal detachment, haemorrhage, lipid, drusen, or choroidal neovascularisation.

Most patients had frequent follow-up examinations with repeat fundus photography and fluorescein angiography on at least 1 occasion. In a few instances follow-up information was provided by the patient's referring ophthalmologist.

For purposes of analysis eyes were separated into 3 groups based on the age of the patient and the presence of choroidal neovascularisation at the time of the initial examination. Group 1 (nonsenile) consisted of eyes from patients under 50 years of age. None had angiographic evidence of choroidal

Table 1 Data from 50 eyes with pigment epithelial detachment

\begin{tabular}{|c|c|c|c|}
\hline & Group 1 & Group 2 & Group 3 \\
\hline & Nonsenile & $\begin{array}{l}\text { Senile, } \\
\text { avascular }\end{array}$ & $\begin{array}{l}\text { Senile, } \\
\text { vascular }\end{array}$ \\
\hline No. of eyes & 14 & 17 & 19 \\
\hline Right:left & $5: 9$ & $6: 11$ & $11: 8$ \\
\hline No. of pts. & 11 & 13 & 18 \\
\hline Male:female & $9: 2$ & $6: 7$ & $11: 7$ \\
\hline Avg. age and range & $43(37-47)$ yr & $64(53-76)$ yr & $67(55-82)$ yr \\
\hline Avg. follow-up (mo) & $\begin{array}{l}23 \\
\text { (range 8-51) }\end{array}$ & $\begin{array}{l}35 \\
\text { (range 14-86) }\end{array}$ & $\begin{array}{l}29 \\
\text { (range 6-75) }\end{array}$ \\
\hline \multicolumn{4}{|l|}{ Initial examination: } \\
\hline $\begin{array}{l}\text { Size of detachment } \\
\text { (disc diameters) }\end{array}$ & $1 / 3$ to 1 & $1 / 2$ to 3 & $1 / 2$ to 3 \\
\hline $\begin{array}{l}\text { Neovascularisation } \\
\text { (eyes) }\end{array}$ & 0 & $\mathbf{0}$ & 19 \\
\hline Drusen (eyes) & 1 & 15 & 19 \\
\hline Haemorrhage (eyes) & $\mathbf{0}$ & $\mathbf{0}$ & 4 \\
\hline Lipid (eyes) & 0 & $\mathbf{0}$ & 9 \\
\hline $\begin{array}{l}\text { Neuroepithelial } \\
\text { detachment (eyes) }\end{array}$ & 3 & 6 & 19 \\
\hline \multicolumn{4}{|l|}{ Visual acuity (eyes): } \\
\hline $20 / 20-20 / 40$ & 13 & 14 & 9 \\
\hline $20 / 50-20 / 100$ & 1 & 3 & 5 \\
\hline $20 / 200$-or less & $\mathbf{0}$ & 0 & 5 \\
\hline \multicolumn{4}{|l|}{ Course (eyes): } \\
\hline Flattened & $7(50 \%)$ & $8(47 \%)$ & $\mathbf{0}$ \\
\hline Unchanged & $7(50 \%)$ & $7(41 \%)$ & 5 \\
\hline Haemorrhage/scar & $\mathbf{0}$ & $2(12 \%)$ & 15 \\
\hline New vessels & 0 & $5(24 \%)$ & 19 \\
\hline \multicolumn{4}{|l|}{ Final visual acuity: } \\
\hline $20 / 20-20 / 40$ & $14(100 \%)$ & $9(53 \%)$ & $1(5 \%)$ \\
\hline $20 / 50-20 / 100$ & 9 & $6(35 \%)$ & $4(21 \%)$ \\
\hline $20 / 200$ or less & 9 & $2(12 \%)$ & $14(74 \%)$ \\
\hline $\begin{array}{l}\text { Same or better than } \\
\text { initial vision }\end{array}$ & $14(100 \%)$ & $10(59 \%)$ & $2(11 \%)$ \\
\hline
\end{tabular}

neovascularisation. Group 2 (senile, avascular) was made up of eyes from patients 50 years of age and older, without choroidal neovascularisation. Group 3 (senile, vascular) comprised eyes from patients $\mathbf{5 0}$ years of age and older in which the presence of choroidal neovascularisation was demonstrated by fluorescein angiography.

\section{Results}

GROUP 1 (NONSENILE)

Fourteen eyes of 11 patients were followed up for 8 to 51 months (average, 23 months) (see Table 1). Nine patients were male and 2 female. The age range was 37 to 47 years (average, 43 years). Five were right eyes and 9 were left eyes. The size of the pigment epithelial detachment varied from onethird disc diameter to 1 disc diameter. One eye had 3 detachments, 1 eye had 2 detachments. and the remaining eyes had 1 detachment each. One lesion was directly beneath the fovea, and 9 were onefourth disc diameter or more from the fovea. Three eyes had a readily apparent detachment of the overlying sensory retina. Minimal drusen were present in 1 eye, but haemorrhage and lipid were present in no eyes. Initial visual acuity was $20 / 20$ or better in 8 eyes, 20/25-20/30 in 4 eyes, and 20/40 or worse in 2 eyes.

During the follow-up period pigment epithelial detachments flattened spontaneously in 7 eyes, leaving a mild degree of pigment disturbance at the site of the lesion. In the remainder of the eyes the detachments persisted and were accompanied by pigment derangement over the dome of the detachment. Haemorrhage did not occur in any eye, and none developed evidence of choroidal neovascularisation. Visual acuity remained the same or improved in all 14 eyes. Eight eyes had a final vision of $20 / 20$ or better, 4 eyes were $20 / 25$, and 2 eyes were 20/30.

GROUP 2 (SENILE, AVASCULAR)

Seventeen eyes of 13 patients were followed up for 14 to 86 months (average, 35 months). There were 6 males and 7 females. The age range was 53 to 76 years (average, 64 years). There were 6 right eyes and 11 left eyes. One eye had two detachments, and the remainder had 1 each. The size of the pigment epithelial detachment varied from one-half to 3 disc diameters. Nine were 1 to $1 \frac{1}{2}$ to 3 disc diameters. One was 3 disc diameters. The lesion extended beneath the fovea in 12 eyes and was less than onefourth disc diameter from the fovea in 5 eyes. A sensory retinal detachment was present in 6 of the 17 eyes. In all patients drusen were present in the involved and/or fellow eye. The initial visual acuity 


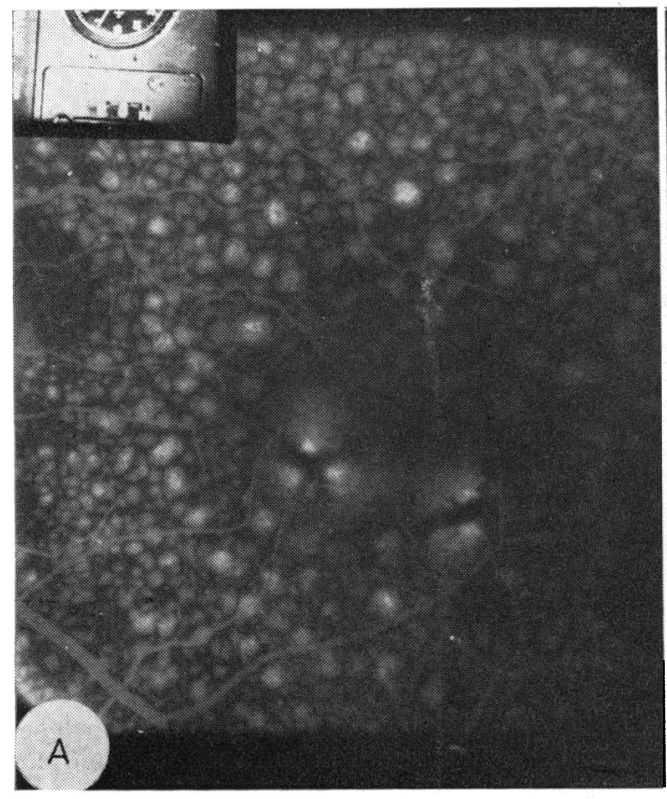

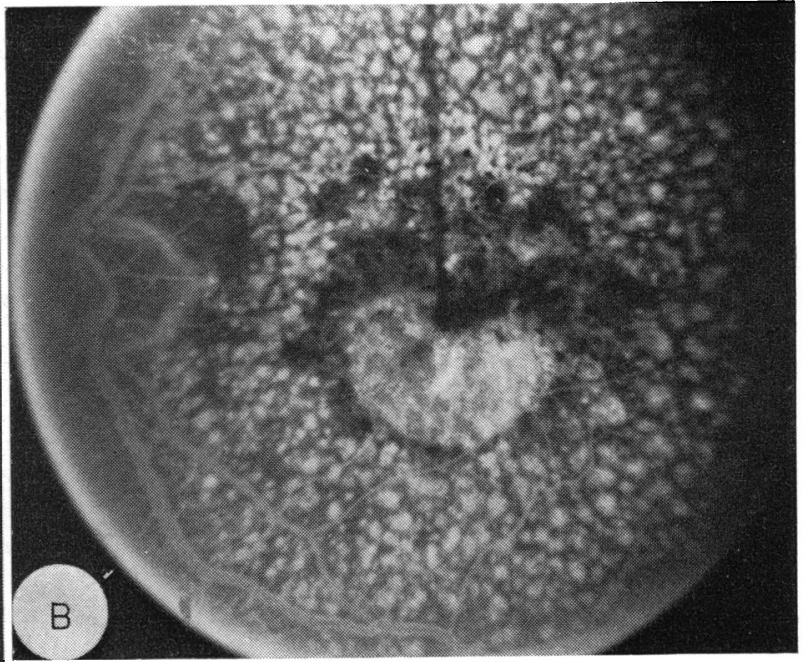

Fig. 1 Case 1. A. Late phase fluorescein angiogram of avascular pigment epithelial detachments with pigment figures in 53-year-old male. Vision =20/30. B. Seven years later. Late phase angiogram shows pigment epithelial atrophy. was $20 / 40$ or better in 14 eyes and $20 / 50$ to $20 / 100$ in 3 eyes.

During the follow-up period the retinal pigment epithelium (RPE) detachment flattened completely in 8 of the 17 eyes, resulting in varying degrees of atrophy of the pigment epithelium (Fig. 1b). In 7 eyes the RPE detachment persisted and was accompanied by progressive pigment disturbance, including the formation of pigment figures over the dome of the detachment. In the remaining 2 eyes the lesion became haemorrhagic, resulting in severe visual loss. In these 2 eyes choroidal neovascularisation was presumed to have developed, but this was not documented with follow-up fluorescein angiography. In 3 other eyes neovascularisation was present on follow-up angiography. In 1 eye this occurred beneath a persistent pigment epithelial detachment, and in 2 eyes it developed temporal to the detachment, which had spontaneously flattened (Fig. 2).

Visual acuity deteriorated (2 lines or more on the Snellen chart) in 7 of the 17 eyes $(41 \%)$ over the study period. Final vision was $20 / 40$ or better in 9 eyes, $20 / 50$ to $20 / 100$ in 6 eyes, and 20/200 or worse in 2 eyes. Of 6 eyes with large detachments $(2$ disc diameters or more) 3 eyes had a final vision of 20/40 or better, 1 eye was 20/60, and 2 eyes were worse than $20 / 200$.

GROUP 3 (SENILE, VASCULAR)

Nineteen eyes of 18 patients were followed up for 6 to 75 months (average 29 months). There were 11 males and 7 females. The age range was 55 to 82 years (average, 67 years). There were 11 right eyes and 8 left eyes. All eyes had subpigment epithelial neovascularisation demonstrated by fluorescein angiography, and were accompanied by a detachment of the sensory retina. Haemorrhage was present in 4 eyes. Neovascularisation was located beneath the fovea in 7 eyes. Drusen were present in the involved and/or fellow eye in all cases. Lipid was noted beneath the retina in 9 eyes. Initial visual acuity was $20 / 20$ to $20 / 40$ in 9 eyes, $20 / 50$ to $20 / 100$ in 5 eyes, and $20 / 200$ or less in 5 eyes.

During the study period increasing haemorrhage and/or scar formation occurred in 15 of the 19 eyes. Final visual acuity was $20 / 40$ or better in one eye, $20 / 40$ to $20 / 100$ in 4 eyes, and $20 / 200$ or less in 14 eyes.

\section{Discussion}

There is only minimal information describing the clinical course of eyes with untreated pigment epithelial detachments. Lewis ${ }^{6}$ reported follow-up data from 32 eyes of 21 patients with untreated serous detachments of the pigment epithelium. All patients were under 55 years of age (average age, 45 years), and there was no accompanying evidence of senile macular degeneration or other ocular disease. $97 \%$ of the eyes had final visual acuity of $20 / 50$ or better after an average follow-up period of 7 years. Similarities in the characteristics and the relatively benign clinical course of this condition to those of central serous choroidopathy were noted. 

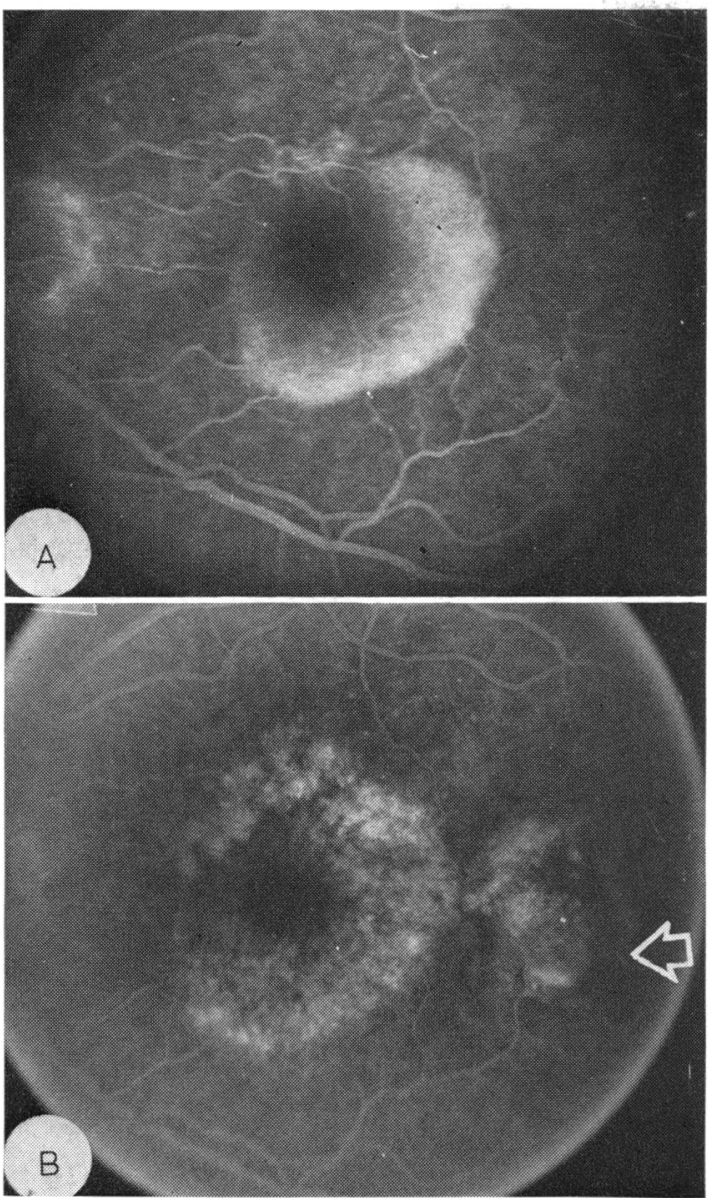

Fig. 2 Case 2. A. Late phase angiogram of avascular pigment epithelial detachment in 71-year-old male.

Vision $=20 / 20$. B. Two years later. Late phase angiogram shows flat retinal pigment epithelium atrophy in area of previous detachment with haemorrhagic subretinal neovascular lesion temporally (arrow). Vision $=20 / 25$.

Gass $^{2}$ observed 7 older patients with serous pigment epithelial detachments of 1-2 disc diameters accompanied by drusen. He reported that without treatment these patients maintained visual acuity for periods of up to 2 years. Teeters and Bird,? however, in their study of untreated senile eyes reported a poor visual prognosis. They found that $61 \%$ of eyes with avascular lesions had a final vision of $6 / 60$ or less. In eyes with vascular lesions the visual outcome was worse.

We studied pigment epithelial detachments in eyes of young adults as well as those occurring in older individuals.

In Group 1 (nonsenile), all patients were under 50 years of age and displayed essentially no accom- panying fundus pathology. The lesions were small (1 disc diameter or less) and initial visual impairment was minimal. During the course of the study period the detachments either flattened spontaneously $(50 \%)$ or remained elevated. None developed choroidal neovascularisation or haemorrhage. The final vision was $20 / 30$ or better in all eyes.

As noted by Lewis, ${ }^{6}$ this group may well be considered a form of central serous choroidopathy in which the pigment epithelial detachment, rather than an overlying sensory retinal detachment, is the predominant feature. Indeed, 3 eyes showed an accompanying sensory retinal detachment, each of which resolved during the course of the study period. The male to female ratio of 9:2, and the self-limited nature of the lesion, with minimal permanent visual loss, is similar to that found in patients with classical central serous choroidopathy ${ }^{8-10}$

In group 2 (senile, avascular) the natural course was variable, and significant visual impairment sometimes occurred. All patients were over 50 years of age, and all eyes displayed drusen, a recognised precursor of further senile macular degenerative changes. ${ }^{211}$

Teeters and Bird ${ }^{7}$ reported 18 eyes with avascular disciform lesions studied with fluorescein angiography, and followed up for four to 19 months (average, 10 months). During this period 12 eyes $(67 \%)$ developed angiographic evidence of neovascularisation. Complete resolution of the detachment occurred in only 3 eyes $(16 \%)$. Eleven eyes $(61 \%)$ had deteriorated to a final vision of 6/60 or worse.

Our findings, representing data from 17 eyes with avascular detachments, followed up for an average of 35 months, produced a better prognosis. Five eyes $(29 \%)$ developed evidence of choroidal neovascularisation, while 2 eyes $(12 \%)$ deteriorated to $20 / 200$ or less during the study.

These conflicting results probably arise from differences in interpretation of choroidal neovascularisation of the fluorescein angiogram. In each of the 17 eyes studied by us the pigment epithelium detachment filled with dye in a nearly uniform and complete fashion. This was not true in some of the eye studies by Teeters and Bird. ${ }^{7}$ In their description of the avascular process lesions were included which, on fluorescein angiography, showed irregular filling and uneven late hyperfluorescence. ${ }^{72}$ We feel that these eyes already had new vessels, and thus the natural course and ultimate visual results were unfavourably influenced. We recognise, however, that new vessels might also be present in eyes which we have classified as avascular with fluorescein angiography. Sarks ${ }^{13}$ has demonstrated the rather common occurrence of choroidal new vessels in senile eyes examined histologically at necropsy, 
even in those without antecedent clinical evidence of their presence.

Our study indicated that avascular pigment epithelial detachments in the senile population differ from those occurring in nonsenile eyes. They tend to be larger, are associated with diffuse degenerative changes in the posterior pole, and in some instances may progress to a neovascular process with possible haemorrhagic sequelae. Those that flattened spontaneously $(47 \%)$ resulted in varying degrees of pigment epithelial disturbance. In some cases this became progressively more pronounced long after flattening of the lesion. Mild to moderate visual impairment generally occurred in these eyes.

In group 3 (senile, vascular) definite evidence of neovascularisation was demonstrated by fluorescein angiography in all eyes. This course was one of progressive deterioration, with 15 of the 19 eyes experiencing further haemorrhage and/or scar formation. The remaining 4 eyes showed no improvement during the study period. The visual outcome was poor, with 14 of the 19 eyes $(74 \%)$ becoming legally blind. These poor results are similar to those reported by Teeters and Bird. ${ }^{7}$ They contrast markedly with the excellent visual prognosis found in avascular detachments in nonsenile eyes (group 1 ), and the variable outcome in avascular detachments in senile eyes (group 2).

It is of interest to consider the role of photocoagulation in the light of these findings.

With regard to the eyes in group 1 (nonsenile), it would seem that similar guidelines to those recommended for the treatment of central serous choroidopathy are indicated. ${ }^{10}$ Unless further benefits become established, photocoagulation should be considered only for those patients in whom visual impairment is disabling or annoying, assuming the lesion is located a safe distance from the fovea.

The value of photocoagulation for eyes in group 2 (senile, avascular) remains uncertain. Bird ${ }^{4}$ reported the flattening of 21 of 24 avascular detachments following photocoagulation. Visual results were relatively good, with $67 \%$ of these eyes achieving $6 / 12$ vision, while none were $6 / 60$ or worse after an average 10 -month follow-up period. Gass, ${ }^{2}$ however, noting that most detachments could be flattened with photocoagulation, found visual results to be variable.

Considering the poor visual prognosis of eyes in group 3 (senile, vascular) one is tempted to consider such eyes for photocoagulation, with heavy focal treatment applied to extrafoveal neovascular membranes. Bird ${ }^{4}$ has reported encouraging results by employing heavy applications of argon laser energy to neovascular membranes as close as one-eighth disc diameter from the fovea. However, because these eyes have diffuse involvement of the choroid, Bruch's membrane, and pigment epithelium, photocoagulation, even if successful, may be of only temporary benefit.

\section{Conclusion}

The clinical course of untreated retinal pigment epithelial detachment varies with the age of the patient and the presence of choroidal neovascularisation. In nonsenile eyes the visual prognosis is excellent. In senile eyes with avascular lesions the visual outcome is variable, with approximately half of all eyes experiencing moderate to severe visual impairment. In senile eyes with vascular lesions the visual prognosis is poor.

Photocoagulation is seldom indicated for lesions in nonsenile eyes. Its value in the treatment of senile eyes is unproved and could best be determined by a prospective, randomised clinical trial. Such a trial is now in progress in the United Kingdom.

\section{References}

${ }^{1}$ Gass JDM. Pathogenesis of disciform detachment of the neuroepithelium. II. Idiopathic central serous choroidopathy. Am J Ophthalmol 1967; 63: 573-85.

${ }^{2}$ Gass JDM. Drusen and disciform macular detachment and degeneration. Arch Ophthalmol 1973; 90: 206-17.

${ }^{3}$ Schatz H, Patz A. Exudative senile maculopathy: I. Results of argon laser photocoagulation. Arch Ophthalmol 1973; 90: 183-96.

${ }^{4}$ Bird AC. Recent advances in the treatment of senile disciform macular degeneration by photocoagulation. Br J Ophthalmol 1974; 58: 367-76.

${ }^{5}$ Gass JDM. Choroidal neovascular membranes-their visualization and treatment. Trans Am Acad Ophthalmol Otolaryngol 1973; 77: 310-20.

${ }^{6}$ Lewis ML. Idiopathic serous detachment of the retinal pigment epithelium. Arch Ophthalmol 1978; 96: 620-4.

'Teeters VW, Bird AC. The development of neovascularization of senile disciform macular degeneration. $A m J$ Ophthalmol 1973; 76: 1-18.

${ }^{8}$ Gass JDM. Pathogenesis of disciform detachment of the neuroepithelium. II. Idiopathic central serous choroidopathy. Am J Ophthalmol 1967; 63: 587-615.

${ }^{9}$ Watzke RE, Burton TC, Leaverton PE. Ruby laser photocoagulation therapy of central serous choroidopathy. Trans Am Acad Ophthalmol Otolaryngol 1974; 78: 205-11.

${ }^{10}$ Klein ML, VanBuskirk EM, Friedman E, Gragoudas E, Chandra S. Experience with nontreatment of central serous choroidopathy. Arch Ophthalmol 1974; 91 : 247-50.

${ }^{11}$ Gragoudas ES, Chandra SR, Friedman E, Klein ML, VanBuskirk M. Disciform degeneration of the macula. II. Pathogenesis. Arch Ophthalmol 1976; 94: 755-7.

${ }^{12}$ Teeters VW, Bird AC. A clinical study of the vascularity of senile disciform macular degeneration. Am J Ophthalmol 1973; 75: 53-65.

${ }^{13}$ Sarks SE. New vessel formation beneath the retinal pigment epithelium in senile eyes. Br J Ophthalmol 1973; 57: 961-5. 